\title{
Medical applications of Terahertz Imaging: a Review of Current Technology and Potential Applications in Biomedical Engineering
}

\author{
K. Humphreys ${ }^{1}$, J. P. Loughran ${ }^{2}$, M. Gradziel ${ }^{2}$, W. Lanigan ${ }^{2}$, T. Ward ${ }^{1}$, J.A. Murphy ${ }^{2}$, C. O'Sullivan ${ }^{2}$ \\ ${ }^{1}$ Department of Electronic Engineering, National University of Ireland, Maynooth, Co. Kildare, Ireland. \\ ${ }^{2}$ Department of Experimental Physics, National University of Ireland, Maynooth, Co. Kildare, Ireland.
}

\begin{abstract}
Terahertz (THz) imaging is in its early stages of development but already the potential clinical impact of this new imaging modality is clear. From cancer research to DNA analysis THz technology is improving or even making possible imaging of hitherto inaccessible phenomena. In this paper we present a short review of $\mathrm{THz}$ imaging from the point of view of biomedical engineering. We discuss the current state of the art in terms of $\mathrm{THz}$ imaging systems; describe current applications, future potential and our own approaches to harnessing this novel technology. We draw attention to open problems in the area with respect to the limitations of the technology before concluding with descriptions of our future work in the area.
\end{abstract}

Keywords - Biosensors, Terahertz Imaging, Tissue Spectroscopy.

\section{INTRODUCTION}

Terahertz $(\mathrm{THz})$ radiation, or 'T-Rays', in its broadest description refers to the region of the electromagnetic spectrum between $100 \mathrm{GHz}$ and $30 \mathrm{THz}$ (wavelengths of 3 $\mathrm{mm}$ to about $1 \mu \mathrm{m})$. This encompasses the region from just beyond microwaves through the far infrared and some way into mid infrared. In the past, generating $\mathrm{THz}$ radiation required bulky and expensive equipment like free electron lasers or alternatively the use of thermal sources to produce weak, incoherent radiation. Detecting $\mathrm{THz}$ radiation was not much easier, requiring liquid helium cooled bolometers with poor noise performance. Indeed the lack of viable sources and detectors lead to this band of the spectrum being referred to as the 'THz Gap'. In recent times however, advances in technology have made possible the production and detection of $\mathrm{THz}$ radiation with solid-state devices operating at room temperature [1, 2]. In so doing, a previously unavailable region of the spectrum has been made accessible, and it is a region of great potential for medical science in particular. While other portions of the spectrum are already well established in medical applications, the properties of the $\mathrm{THz}$ band allow it to occupy a new niche. THz quanta are far less energetic than those of x-rays and pose no ionization hazard for biological tissue. While this is also true of microwaves, the shorter wavelengths of the $\mathrm{THz}$ band allow for greater spatial resolution.

The authors would like to acknowledge the financial support of Science Foundation Ireland and the Irish Research Council for Science, Engineering and Technology.

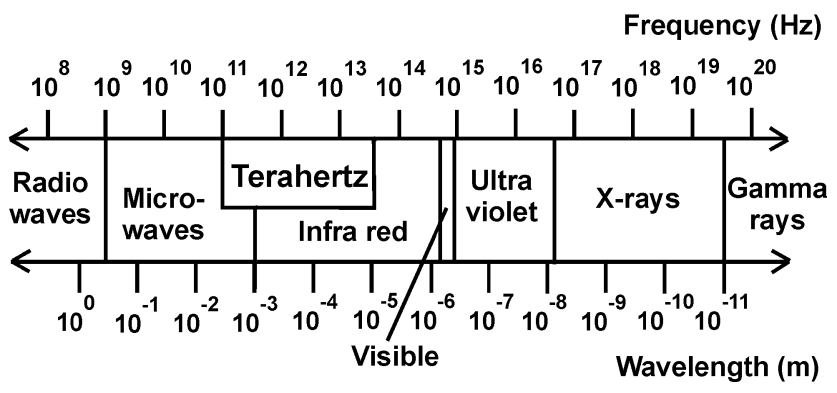

Fig. 1. The THz waveband.

When interacting with heterogeneous biological tissue, the comparatively long $\mathrm{THz}$ wavelengths can penetrate much further than visible light or the near infrared - the longer $\mathrm{THz}$ wavelengths being less susceptible to effects such as Rayleigh scattering. Furthermore, $\mathrm{THz}$ radiation excites rotational and vibrational modes of some biological molecules and $\mathrm{THz}$ radiation has already been shown to have good tissue differentiating abilities [3].

We are in the midst of a revolution in noninvasive imaging technologies for biomedical science and optical/quasi-optical techniques are forming the vanguard in this area. $\mathrm{THz}$ technology is well placed to provide the impetus for the development of the next wave of noninvasive biomedical instruments and it is important that biomedical engineers and scientists embrace this technology early in its development. To this end, this paper is an exposition of $\mathrm{THz}$ imaging technology with an emphasis on its current applications and future directions. Our purpose here is to prime the field of biomedical engineering for this increasingly relevant technology. The layout of the paper is as follows. Section II briefly summarizes technologies required to generate and detect T-Rays. The current range of imaging applications in medicine and biology is largely determined by the absorption of T-Rays by water. In section III we give a concise account of these applications. Section IV discusses shortcomings of the technology and techniques used to address these. In Section $\mathrm{V}$ we propose some improvements to quasi-optical design of delivery and measurement systems that will allow $\mathrm{THz}$ to attain its true potential in a clinical environment. We also include a description of a proposed experimental $\mathrm{THz}$ Fourier optics system for probing theoretical concepts. We conclude with a brief synopsis of the future viability of the technology in the biomedical field as well as describe some of the open problems in the area. 


\section{GENERATING AND DETECTING THZ RADIATION}

While there are numerous ways of generating $\mathrm{THz}$ radiation, most of the useful sources and detectors can be divided into two groups - those based on electronics and those based on photonics [4]. The electronic sources, which include Gunn diodes, backward wave oscillators (BWO) and super-lattice electronic devices, for example, are extensions of high frequency electronics and as such can only operate at the microwave end of the $\mathrm{THz}$ region. The upper frequency limit for electronic sources is approximately $300 \mathrm{GHz}$ (up to $1 \mathrm{THz}$ for a BWO) and they usually provide limited power at $\mathrm{THz}$ frequencies. Their use is not currently widespread in medical applications.

By comparison, photonic sources - lasers, photoconductive dipole antennas (PDA), and devices relying on optical mixing to generate $\mathrm{THz}$ radiation - are ubiquitous in medical applications. They are used to provide both narrowband continuous wave $(\mathrm{CW})$ radiation and broadband $\mathrm{THz}$ pulses across a wide range of frequencies.

Lasers, although viable, are still uncommon as a primary source of $\mathrm{THz}$ radiation in medical applications, owing to the cost, complexity and indeed size of the devices currently available. It is often easier to generate $\mathrm{THz}$ radiation by optically mixing light from two sources; typically two laser diodes, whose difference in frequency is in the $\mathrm{THz}$ range. Other methods can be used if pulsed operation is acceptable or, indeed, required. Photoconductive dipole antennas can be used to produce broadband $\mathrm{THz}$ pulses up to about 1 THz. Perhaps the most commonly used generation method, in medical applications, employs optical rectification, whereby the high frequency oscillations of a femtosecond laser pulse are rectified by an optical crystal, leaving only the envelope of the laser signal - which is a THz pulse [4].

It is worth noting that both PDA and optical rectification based sources require the use of a femtosecond pulsed laser, operating at a wavelength in the region of $800 \mathrm{~nm}$. This adds considerably to the cost of these sources.

As far as detection is concerned, one usually wants to employ a coherent detection scheme, so that both the amplitude and the phase of the $\mathrm{THz}$ field can be captured. Both PDA and optical rectification methods can be used to that end.

Another notable detector type is based on an electrooptic effect, where the presence of a $\mathrm{THz}$ frequency electric field induces a birefringence in some optically transparent material. The magnitude of this effect, and thus the state of the $\mathrm{THz}$ field, can be ascertained by analyzing the change of the polarization states of fast optical pulses transmitted through the material [5].

In summary, the technology now exists to generate and detect coherent $\mathrm{THz}$ radiation at useful power levels, either as a continuous wave or as a series of pulses.

\section{CURRENT AND FUTURE USES OF THZ RADIATION}

$\mathrm{THz}$ radiation has found two fundamental modes of application in medicine and biology - spectroscopy and imaging, though far more use has been made of the latter in medical applications. Here we will limit our discussion to imaging, though it is worth noting that $\mathrm{THz}$ time-domain spectroscopy has been shown to be capable of detecting mutations and conformational modes of biomolecules provided a reference sample is available for calibration [6]. In addition, T-rays have been used as a method of label-free probing of the binding state of DNA with good results $[7,8]$.

An unavoidable issue when imaging with $\mathrm{THz}$ radiation is absorption by water. The entire $\mathrm{THz}$ band is strongly absorbed by water (or any polar liquid) [9] and consequently does not penetrate moist tissue to any significant depth. It has been reported that a $\mathrm{THz}$ pulse can be detected through $1.5 \mathrm{~mm}$ of skin when the signal to noise ratio is 500:1 [10], this is low penetration but still better than through water alone $[3,9]$. Researchers have circumvented this in two ways, firstly by performing transmission imaging on thin, clinically prepared tissue samples, and secondly by imaging surface features using reflection geometry imaging. Transmission imaging, for obvious reasons, can only be carried out in vitro, whereas a reflection geometry set-up allows the possibility of in vivo imaging $[11,12]$.

The limited penetration depth of $\mathrm{THz}$ radiation has focused medical imaging research into the areas of dermatology and dentistry. In dentistry, in vitro experiments using $\mathrm{THz}$ imaging and spectroscopy have already been carried out to determine the characteristic $\mathrm{THz}$ properties of enamel and dentine in human teeth [13]. $\mathrm{THz}$ radiation has been shown to be capable of the early detection of dental carries [14]. Since in vivo $\mathrm{THz}$ imaging is currently limited to surface features, it would seem that dentistry should be a suitable field of application. However, in practice $\mathrm{THz}$ imaging systems are large and cumbersome - even structures as obvious as teeth can make a challenging target. In this respect $\mathrm{THz}$ imaging is still some way off offering a non-ionizing alternative to x-rays in dentistry.

Imaging with $\mathrm{THz}$ radiation has been most successful when dealing with illnesses of the skin. Work in this area initially focused on identifying previously diagnosed basal cell carcinomas (a cancer of the lowest layer of the epidermis). At first this was accomplished in vitro using both transmission and reflection modes [11, 15, 16], where $\mathrm{THz}$ showed promising tissue differentiating abilities being able to distinguish between diseased and normal but inflamed tissue. More recently reflection geometry techniques have been used to take the first in vivo $\mathrm{THz}$ images of skin cancers, showing surface features and depth information [17]. In both the in vivo and in vitro tests, $\mathrm{THz}$ radiation has been shown to perform well when compared to the standard in vitro histological test. 
The potential usefulness of $\mathrm{THz}$ imaging in this case cannot be over stated. The treatment for this type of cancer is surgical removal of the tumor, the object being, to remove as little healthy tissue as possible while ensuring all cancerous material has been removed. Histological examination of the excised tissue indicates whether the tumor has been successfully removed. THz imaging allows the size, shape and depth of the diseased tissue to be accurately viewed prior to surgery.

The exact reason for $\mathrm{THz}$ radiation's ability to differentiate between cancerous and healthy tissue is not known. While one might suspect that water absorption may play a part in the in vivo cases - water is removed from samples prior to histological testing and on such samples $\mathrm{THz}$ still performs well. Since cancerous and healthy tissue do not differ greatly in density, it has been suggested that the cause of the differentiation is down to chemical composition [18].

Another area where $\mathrm{THz}$ imaging will likely find use is wound assessment. The potential of $\mathrm{THz}$ imaging as a burn diagnostic has been demonstrated using chicken breast [3, 19]. It is also conceivable that $\mathrm{THz}$ imaging could be of use in monitoring treatment of skin conditions (like psoriasis), since $\mathrm{THz}$ imaging is cheaper than MRI and does not necessitate contact with the skin like ultrasound. (See [20] and the references therein.)

\section{PROBLEMS FACING THZ USE IN MEDICAL APPLICATIONS}

In addition to the problem of water absorption (which may in certain circumstances be turned to our advantage), several issues need to be addressed so that $\mathrm{THz}$ radiation may be used to its full potential in medicine.

\section{A. Acquisition Speed and Signal to Noise Ratio}

When compared to established medical imaging modalities $\mathrm{THz}$ imaging is a slow process. The fastest systems boast acquisition rates of thousands of pixels per second while the slowest take several seconds to acquire each pixel. Since sources are expensive, it is usual for an imaging system to employ only one, meaning that the sample or detector must be mechanically moved about in a scanning pattern. As an alternative, in $\mathrm{CW}$ systems the radiation can be shaped into a beam that covers the whole sample at once and an array of detectors used, however this severely impinges on the signal-to-noise ratio (SNR). A real-time $\mathrm{THz}$ imaging system can be realized using an electro-optic detector in conjunction with a CCD camera. Unfortunately, the use of the CCD camera precludes the use of phase sensitive detection techniques, which decreases the SNR by several orders of magnitude [5].

\section{B. Resolution}

The resolution of any conventional imaging system is diffraction limited by the wavelength of the radiation. In the case of $\mathrm{THz}$ imaging this corresponds to features in the range $1 \mu \mathrm{m}$ to $3 \mathrm{~mm}$, not detailed enough for many medical applications. There are however ways to overcome this and the foremost among them is near-field imaging. Near-field imaging is based on the principle that the distribution of radiation directly behind a small aperture is a function of the diameter of the aperture rather than a function of wavelength or diffraction. When the object to be imaged is placed close to the aperture (less than a wavelength separation), a spatial resolution of a fraction of a wavelength $(\lambda)$ can be achieved. Resolutions of better than $\lambda / 4$ have been demonstrated [21].

The drawback to this technique is the proximity in which the sample must be placed to the aperture. A distance of less than $1 \mathrm{~mm}$ is required for frequencies of $300 \mathrm{GHz}$ and above, which makes the technique impractical for in vivo reflection mode use. The technique is however still valuable for in vitro imaging on prepared samples.

Other researchers have developed a technique that combines $\mathrm{THz}$ images taken over different frequency intervals to display details in histological samples that are not visible under normal microscopic inspection [18]. The technique does however require some image processing to mitigate the artifacts introduced by combining images of different resolutions (the resolution being related to the wavelength used).

\section{Tissue Differentiation and a Spectral Database}

One piece of research that would greatly benefit $\mathrm{THz}$ imaging, sensing and spectroscopy, is the compilation of a database detailing the response of biological tissues within the THz band. Some such data have already been published in the case of DNA [22], while the transmission, refractive index and absorption coefficients of several human tissues have been documented in the frequency range 0.5 to 1.5 $\mathrm{THz}[23]$.

\section{NOVEL OPTICAL DESIGN FOR THZ IMAGING APPLICATIONS}

There are a number of issues for $\mathrm{THz}$ imaging which result from the limitations of the long wavelength of the radiation and the necessity to use efficient quasi-optical techniques in designing such systems. We therefore are in the process of developing a long wavelength Fourier Optics set-up for the analysis of THz images (see Fig. 2). Initially the set-up is allowing us to investigate the far-infrared optical characteristics of various organic materials and biomedical tissues. We are particularly interested in determining differences in the absorption and reflecting properties related to variation in the water content. The Fourier set-up is equivalent to a Gaussian Beam telescope as usually described in quasi-optics and so can also be used for imaging of a sample when it is placed at the input focal plane (see Fig. 2(B)). This offers the opportunity of applying spatial filtering techniques at the intermediate Fourier plane (between the two lenses), and of extending traditional visible wavelength techniques to the far infrared, where 
spatial resolution and image quality will be a big issue. We also intend to model these effects computationally and apply image processing based on a modal approach that is thus optimized for the unique low throughput optics.

We have also been probing the development of novel beam forming components for the specialized requirements of $\mathrm{THz}$ imaging systems. For example very useful pencil like Bessel beams produced by axicons have the possibility of giving high resolution and large depth of field [24]. In a bid to improve further the usefulness and cost of such components in a practical optical set-up we are now investigating binary versions of these components in combination with binary lenses etc. The components can be studied theoretically using powerful quasi-optical approaches based on Gaussian mode analysis. Another area we intend to develop is that of flexible waveguide delivery systems based on corrugated waveguides. Such systems are difficult to model efficiently, but we have developed powerful tools to allow us to do this. Our aim is to pioneer novel optical techniques, which are appropriate for both the unique long wavelength characteristics of $\mathrm{THz}$ and millimetre-waves, and reduce the cost of manufacture compared with typical existing customized designs.

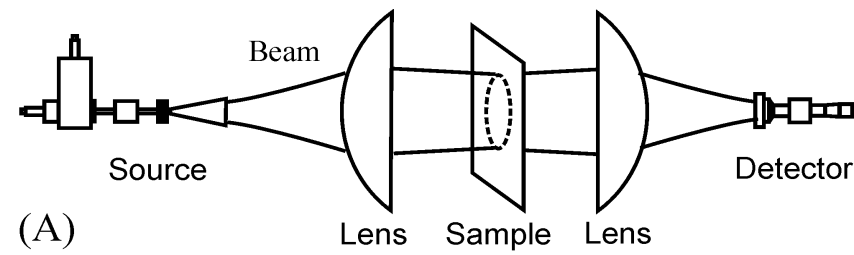

(B)

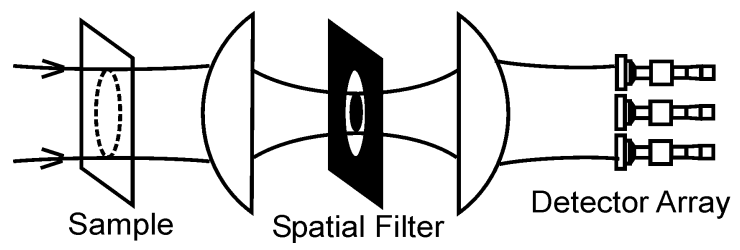

Fig. 2. Long-wavelength Fourier optics system showing (A) the sample characterization and (B) the imaging configuration.

\section{REFERENCES}

[1] Proceedings of 14th International Symposium on Space Terahertz Technology, Tucson, Arizona, 2003.

[2] Proceedings of 11th International Conference on Terahertz Electronics, Sendai, Japan, 2003.

[3] D. M. Mittleman, R. H. Jacobson, and M. C. Nuss, "T-Ray imaging," IEEE Journal on Selected Topics in Quantum Electronics, vol. 2, pp. 679-692, 1996.

[4] S. Mickan, D. Abbott, J. Munch, X.-C. Zhang, and T. van Doorn, "Analysis of system trade-offs for terahertz imaging," Microelectronics Journal, pp. 503-514, 2000.

[5] Z. Jiang and X.-C. Zhang, "Terahertz imaging via electrooptic effect," IEEE Transactions on microwave theory and techniques, vol. 47, pp. 2644-2650, 1999.

[6] A. Markelz, S. Whitmire, J. Hillebrecht, and R. Birge, "THz time domain spectroscopy of biomolecular conformational modes," Physics in Medicine and Biology, vol. 47, pp. 3739-3805, 2002.
[7] P. Haring Bolivar, M. Bruchereifer, M. Nagel, H. Kurz, A. Bosserhoff, and R. Buttner, "Label-free probing of genes by timedomain terahertz sensing," Physics in Medicine and Biology, vol. 47, pp. 3815-3821, 2002.

[8] M. Nagel, P. Haring Bolivar, M. Brucherseifer, and H. Kurz, "Integrated THz technology for label-free genetic diagnostics," Applied Physics Letters, vol. 80, pp. 154-156, 2002.

[9] L. Thrane, R. Jacobsen, P. Uhd Jepsen, and S. Keiding, "THz reflection spectroscopy of liquid water," Chemical Physics Letters, vol. 240, pp. 330-333, 1995.

[10] C. D. Benzant, "Applications of THz pulses in semiconductor relaxation and biomedical imaging studies." PhD Thesis: University of Nottingham, Nottingham, U.K., 2000.

[11] R. H. Woodward, V. P. Wallace, and R. J. Pye, "Terahertz pulse imaging in reflection geometry of skin tissue using time domain analysis techniques," Proc. SPIE, vol. 4625, pp. 160-169, 2002.

[12] R. H. Woodward, B. Cole, V. P. Wallace, D. D. Arnone, E. H. Linfield, and M. Pepper, "Terahertz pulsed imaging in reflection geometry of human skin cancer tissue," Physics in Medicine and Biology, vol. 47, pp. 3853-3863, 2002.

[13] N. Zinov'ev, A. Fitzgerald, S. Strafford, D. Wood, F. Carmichael, R. Miles, et al., "Identification of tooth decay using terahertz imaging and spectroscopy," presented at Twenty Seventh International Conference on Infrared and Millimeter Waves, 2002.

[14] C. M. Ciesla, D. D. Arnone, A. Corchia, D. Crawley, C. Longbottom, E. H. Linfield, et al., "Biomedical applications of terahertz pulse imaging," Proc. SPIE, vol. 3934, pp. 73-81, 2000.

[15] R. H. Woodward, B. Cole, V. P. Wallace, D. D. Arnone, R. Pye, E. H. Linfield, et al., "Terahertz pulse imaging of in-vitro basal cell carcinoma samples," presented at Conference on Lasers and Electro Optics, Technical Digest, 2001

[16] R. H. Woodward, V. P. Wallace, R. J. Pye, and e. al, "Terahertz pulsed imaging of ex vivo basal cell carcinoma," Journal of Investigative Dermatology, vol. 120, pp. 72-78, 2003.

[17] A. J. Fitzgerald, V. P. Wallace, R. M. Woodard, E. Pickwell, R. Pye, and D. D. Arnone, "Terahertz pulsed imaging for medical applications," presented at World Congress on Physics and Biomedical Engineering, Sydney, Australia, 2003.

[18] P. Knobloch, C. Schildknecht, T. Kleine-Ostmann, M. Koch, S. Hoffmann, M. Hofmann, et al., "Medical THz imaging: an investigation of histo-pathological samples," Physics in Medicine and Biology, vol. 47, pp. 3857-3884, 2002.

[19] D. M. Mittleman, M. Gupta, R. Neelamani, R. G. Baraniuk, J. V. Rudd, and M. Koch, "Recent advances in terahertz imaging," Applied Physics, vol. B 68, pp. 1085-1094, 1999.

[20] A. J. Fitzgerald, E. Berry, N. N. Zinovev, G. C. Walker, M. A. Smith, and J. M. Chamberlain, "An introduction to medical imaging with coherent terahertz frequency radiation," Physics in Medicine and Biology, vol. 47, pp. 67-84, 2002.

[21] S. Hunsche, M. Koch, I. Brener, and M. C. Nuss, "THz near-field imaging," Optics communications, vol. 150, pp. 22-26, 1998.

[22] M. Brucherseifer, M. Nagel, P. Haring Bolivar, and H. Kurz, "Labelfree probing of the binding state of DNA by time-domain terahertz sensing," Applied Physics Letters, vol. 77, pp. 4049-4051, 2000.

[23] C. D. Sudworth, A. J. Fitzgerald, E. Berry, N. Zinov'ev, S. Vanniasinkam, R. E. Miles, et al., "The Optical Properties of Human Tissue at Terahertz Frequencies," Proc. SPIE, vol. 5143, pp. 24-25, 2003.

[24] N. Trappe, R. Mahon, W. Lanigan, M. J., and S. Withington, "The quasi-optical analysis of Bessel beams in the far-infrared," Infrared Physics \& Technology, In press. 